\title{
An Efficient Method of Image Segmentation for Harvest Time Identification
}

\author{
Monika Bhatnagar \\ Research Scholar, \\ Dr. K. N. Modi \\ University,Rajasthan, India
}

\author{
Prashant Kumar Singh \\ ISC Software Pvt Ltd, Bhopal, \\ India
}

\begin{abstract}
The aim of development and advancements in every research and technology is to provide easy solutions to any user's problems including the day to day ones. One problem that a farmer might face is to identify whether his crop is ready for harvesting or not. Hence the efforts are taken to figure out an easy solution for a farmer where he can use his cellular phone to identify whether his crop is ready for harvesting or not. This he can do by taking picture of his crop through the digital camera of his cellular phone. Therefore image processing plays an active role to figure out the solution to this problem. This paper proposes image segmentation of the image of a crop such as tomato to identify whether the crop is ripe enough or not. The feature that is extracted for image segmentation is moment. The classification technique used for segmentation is K-means clustering algorithm. The values for the segmented image viz. Mismatching Rate, Misclassification Rate, PSNR and MSE are then calculated Then to identify how much the system is robust noise is inserted in the image and then again the image is segmented and the above mentioned values are calculated. Then the image is denoised and again segmentation of the image is performed and the same process is followed. This paper presents the above mentioned process and the system produces equivalent results in all the three cases. The research work presents here the result of the image segmentation done on the image of a tomato using MATLAB version 7.10.
\end{abstract}

\section{Keywords}

Image Segmentation, Moment Based Clustering, Misclassification Rate ,Mismatching Rate, PSNR, MSE

\section{INTRODUCTION}

In today's world technology finds its presence anywhere and everywhere. We find ever progressing advancements in technology in every gazette and software. There are gazettes and software to solve so many day to day problems of life. Like any other advancements we see that one that is coming very rapidly is in the field of image capturing devices such as the cameras. Initially we had cameras for having black and white pictures which then got advanced to get coloured pictures. Then the world of digital cameras came which allowed users to not only have paper form of storing pictures but also storing pictures in digital format. Then, there were advancements in the megapixel values of the camera. Therefore we see that image capturing devices have also not remained untouched by these advancements. One feature that these devices are now used for are that images that they capture now are compared to some other images to get some desired result. Such features find their good use in examples like user authentication using biometric devices. Also a popular use we see are done by the social networking sites where they allow a user to have his or her picture be compared to the celebrities to identify the look-alikes.
This idea can be used and further enhanced to provide an automated solution to farmers to identify the right time of harvesting their crop through some limited device like a cellular phone.

Now a day cellular phones are most common gazette around the world, even amongst Indian Farmers. So assuming that a farmer will have a cellular phone with a good digital camera we are proposing a system wherein a farmer will have an automated solution of identifying the harvest time for his crop on a device he already possesses. To attain this some classification or clustering of image to identify which amongst crop is ripe should be done. To attain this processing of image on the cellular phone needs to be done. In this case to process image, image segmentation of Image mining is used.

Image processing is part of Datamining technique. So to start with the work it is required to understand what the various techniques are that is being dealt with. To start with at first the word Datamining is defined. Datamining may be defined as a process which results into a meaningful information from a given dataset [1]. Datamining finds its use in all the various datasets such as video, image or text. Of all the techniques from datamining here image mining is considered because the work is based on images of crops. Image mining may be defined as a technique that draws an implicit knowledge from datasets made of images and sometimes images and text in combination [2].Image mining technique very well goes with classification techniques or segmentation techniques .In this case segmentation technique is used. Segmentation may be defined as a process of extracting and representing information from an image into group of pixels together based on some measures of similarity [3]. Image classification and image segmentation are the processes that generally go hand in hand. Therefore it can be said that before the classification of an image segmentation s needed. And segmentation is a process that makes use of an image classification algorithm for segmentation process. In this case segmentation will be done on a color image because color of a crop can become a prime concern to identify the time of harvesting.

For any image processing technique, feature extraction is an extremely essential part [4]. The efficiency of any image classifier is dependent on the choice of the feature selected for image processing. Among several sets of features that is available for extraction the feature that is considered for segmentation in this case is moment. Also to analyse the result of segmentation the factors that are taken under consideration are Mismatching Rate (MMR) and Misclassification Rate (MCR), Peak Signal to Noise Ratio(PSNR) and MSE. For segmentation process we are using clustering algorithm.

Clustering is the classification technique which comes under the category of unsupervised learning [5]. The essence of unsupervised learning is that it does not use pre-defined 
training data set for classification. One excellent benefit one can get by using unsupervised classification technique is that the classifier constructed are lightweight classifiers which do not require much space so can be easily implemented on a limited device such as cellular phone. Amongst large number of pixels available clustering groups pixels into classes consisting pixels of similar kind based on some feature considered. Amongst several available clustering algorithms we are using K-Means algorithm to perform the segmentation of the image.

\section{LITERATURE SURVEY}

To provide an automated solution to farmer it was needed to analyse whether classification on the image of a crop such as tomato is possible or not. Therefore it is seen that researchers have been considering tomato and its ripeness a matter of concern for classification.

G. Polder, G. W. A. M. van der Heijden, I. T. Young [6] have shown that spectral images offer more discriminating power than standard RGB images for measuring ripeness stages of tomatoes. Therefore they do let us know that ripeness can be considered for our problem as we want to let a farmer check the ripeness of his crop.

Hassan Asadollahi, Morteza Sabery Kamarposhty, Mir Majid Teymoori [7] have presented in their paper the tomato classification by images processing. So this paper does allow us to look towards the classification of images of crops.

Now the need is to classify image on a cellular phone because this will be most easily available device to an Indian farmer.

Giuseppe Amato, Paolo Bolettieri, Gabriele Costa, Francesco La Torre, Fabio Martinelli have presented a prototype for parental control that detects images with adult content received on a mobile device [8]. This paper in general gives the idea that image classification can be done on cellular phone. The problem with this paper is that the image classification is done on the server side of the cellular phone. So the drawback with this idea is that in case there is any sort of connection failure between the client and server the classification result might not be available at the time of need [8]. Classification should be performed on the device to have better results.

So it is needed to decide which technique is going to be used to build this automated solution. In respect to this it is identified that segmentation is one of the most efficient techniques one can use to identify crop ready for harvest. Ashish Phophalia, Suman K. Mitra, Charu Chawla[3] have shown the illustrated method of moment based image segmentation. They give us the complete idea about the image segmentation and their usage based on a particular set of moment.

\section{PROBLEM STATEMENT}

It is required to identify some method by which one can make a selection between tomatoes ready for harvesting and those which are not ready for harvesting. This would help farmers have an automated solution for their problem.

\section{PROPOSED SOLUTION}

Here K-Means algorithm of clustering is chosen. The reason to choose K-Means algorithm is that unlike most of the clustering algorithm it does not have predefined set of clusters [9]. As the name suggests it can have $\mathrm{K}$ number of clusters. Secondly, at least one item will be present in the clusters and the clusters will not remain empty. Finally, the clusters created will be non-hierarchical and will not overlap. This algorithm is implemented using MATLAB version 7.10. The algorithm is implemented on image with noise and without noise in order to prove the robustness of the algorithm. The consideration for noise was required because many times images acquire the random variation of brightness or colour information in images produced by sensors and circuitry of an image capturing device such as digital camera.

Since time of harvesting is the key issue in this paper therefore moment based segmentation is used wherein moment is considered for the window size selected on an image for segmentation. It was $\mathrm{Hu}$ who proposed that moment could be considered for image processing[10]. Hu's Uniqueness Theorem states that if $\mathrm{f}(\mathrm{x}, \mathrm{y})$ is piecewise continuous and has nonzero values only in the finite part of the $(x, y)$ plane, then geometric moments of all orders exist. It can then be shown that the moment set $\{\mathrm{mpq}\}$ is uniquely determined by $f(x, y)$ and conversely $f(x, y)$ is uniquely determined by $\{\mathrm{mpq}\}$. Since an image segment has finite area and in the worst case, is piecewise continuous a moment set can be computed and used to uniquely describe the information contained in the image segment Among different types of moments existing [3] Statistical moment is used.

Now let us see how classification will be done based on a moment based window. In order to compute moment an overlapping window based method is applied on each pixel. All possible windows are hence defined in which current pixel is placed at different positions of window and moment value is computed for each window representation.

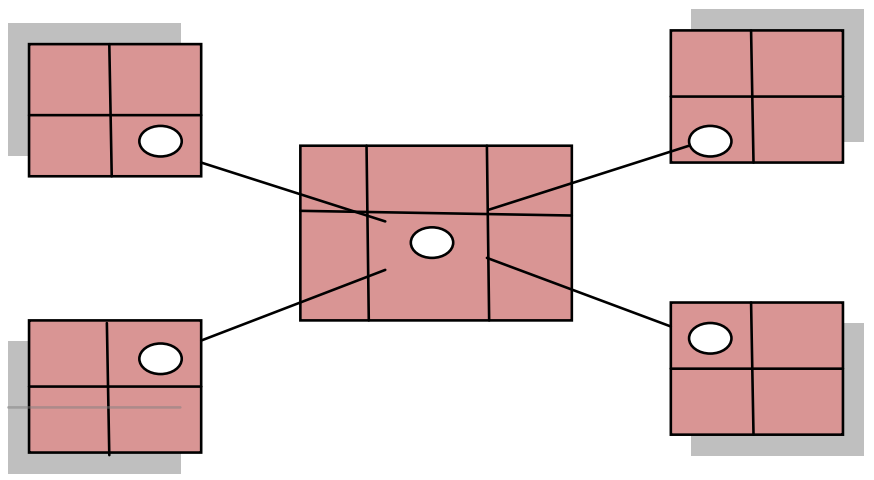

Fig 1:All possible windows for the pixel located in $2 * 2$ Window

There is no specific method to estimate window size which is one of the crucial issues and will be suitable for different types of images and fulfil all requirements. The purpose of considering different window size is to analyze their effects on execution time and various features of image. The window size will also affect the value of moment.

The algorithm proposed for the implementation is as follows:

(a) Choose a highest label $\mathrm{L}$ as number of cluster

(b) Assign a window size $\mathrm{N}^{*} \mathrm{~N}$.

(c) For each pixel in an image find the objects or shapes in an image and label them as $1,2,3 \ldots . . \mathrm{k}$.

(d) For the window compute Statistical Moment values by calculating mean, variance, skewness using pixels of this window. 
(e) Considering the feature vectors consisting mean, variance, skewness, label classify pixel using K-Means classification technique.

(f) The result of this classification is the segmented image.

Finally, four values are calculated viz. MMR, MCR, PSNR and MSE which are explained as

Mismatching Rate $=$ Percentage of labels differing in two abeled window.

Misclassification Rate $=$ (Number of wrongly classified pixels/Total Number of Pixels)*100

Mean Square Error(MSE)

$=\frac{1}{m n} \sum_{i=0}^{m-1} \sum_{j=0}^{n-1}\lfloor I(i, j), K(i, j)\rfloor^{2}$

where, considering a noise-free $\mathrm{m} \times \mathrm{n}$ monochrome image I has its noisy approximation $\mathrm{K}$.

PSNR, may be defined as the ratio between the maximum possible power of a signal and the power of corrupting noise that affects the fidelity of its representation and may be calculated as

PSNR(Peak Signal To Noise Ratio) $=10 \cdot \log _{10} \frac{M A X I^{2}}{M S E}$

Where, Here, $M A X_{I}$ is the maximum possible pixel value of the image

\section{EXPERIMENTAL RESULT}

The algorithm was implemented using MATLAB version 7.10. The images needed for the implementation of the algorithm were captured from a field in Misrod, Bhopal, MP, India.

The input image that was taken in the algorithm is given in Figure 2.

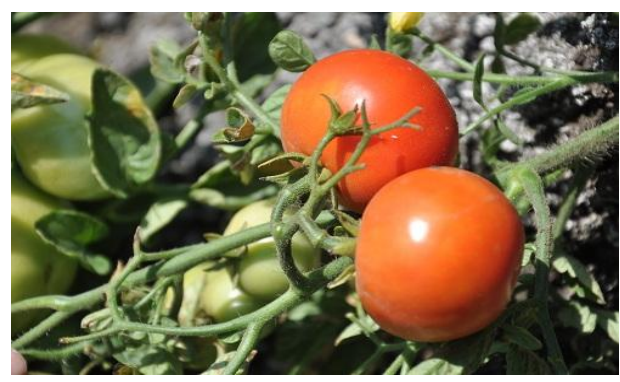

Fig 2:Tomato as input image

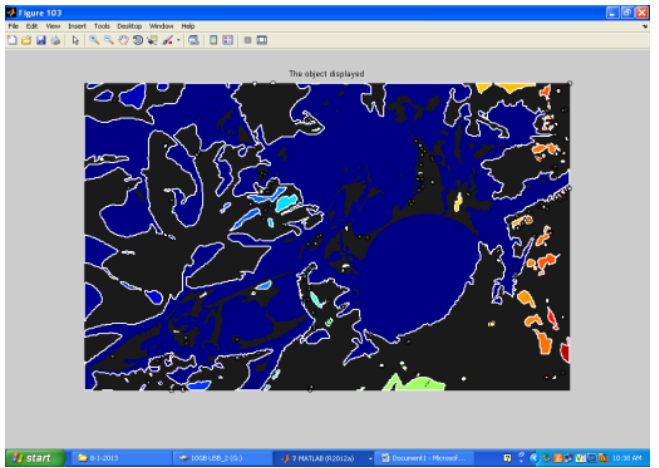

Fig 3. The result of edge detection of the input image
Once the image was taken as input various outputs of images were generated as result of various steps of algorithm. To start with the first image that is generated is the output of image detection as shown in Figure 3. What is seen is the edge detected in the input image. In the next step noise of the type salt and pepper is inserted in the segmented image. Figure 4 shows the image with noise.

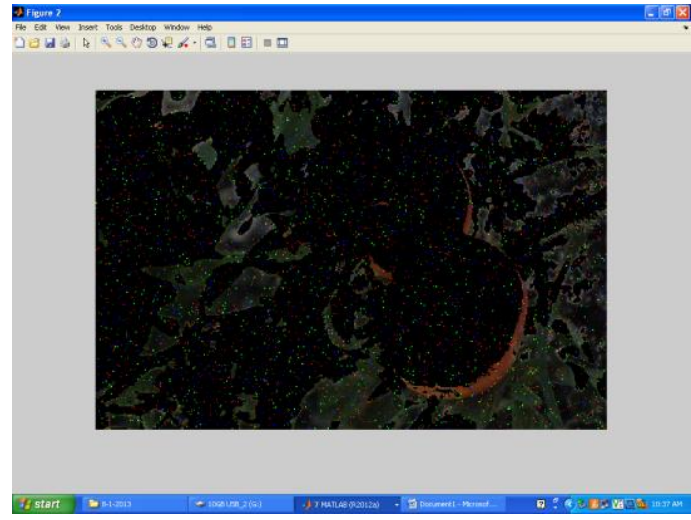

Fig 4: Salt and pepper inserted noise in the segmented image

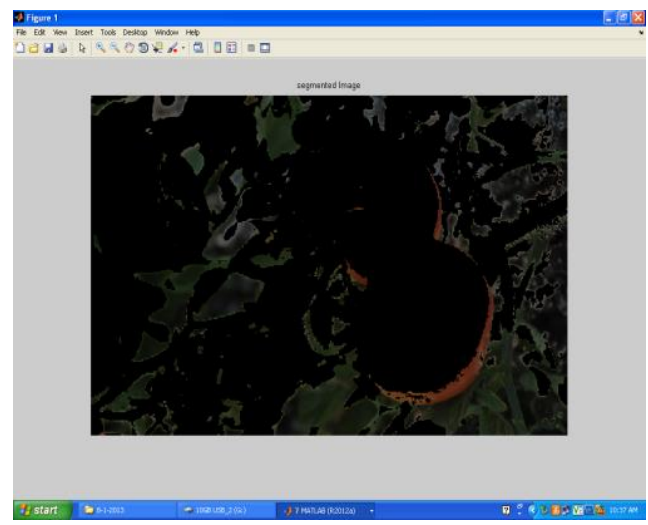

Fig 5: Segmented Image without noise.

Same image when segmented is displayed without noise as shown in Figure 5.

The result of algorithm was also displayed in multiple color. The result is shown in Figure 6.This result is same for image with noise or without noise. The blue part in last image in Figure 6 is clearly the ripe tomatoes.

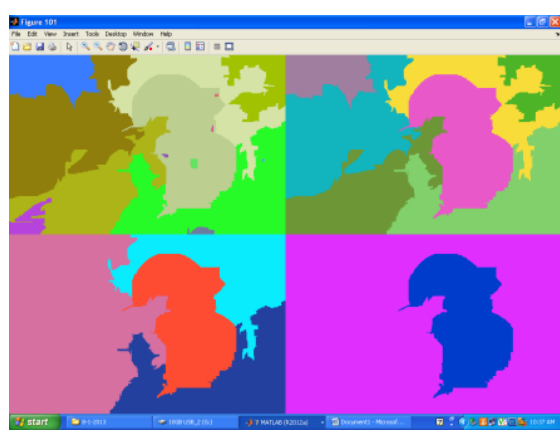

Fig 6: The result of segmentation in multiple colors

To have more clear vision of ripe tomatoes the result of segmentation was also displayed in three colors as shown in Figure 7. Here also the last part of the figure consists of the orange part displaying the ripe tomatoes. 


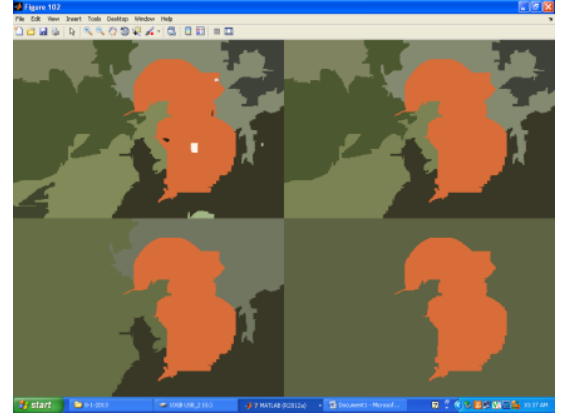

Fig 7:The result of image segmentation in three colors.

Finally, the values of MMR, MCR, MSE and PSNR are calculated which are given Table 1.

Table 1. The final values calculated for the given image.

\begin{tabular}{|l|l|l|l|l|}
\hline $\begin{array}{l}\text { Values } \\
\text { Calculated }\end{array}$ & MMR & MCR & MSE & PSNR \\
\hline With Noise & 1.10 & 0.69 & 28.325 & 148.65 \\
\hline Without Noise & 1.09 & 0.68 & 27.555 & 145.23 \\
\hline
\end{tabular}

\section{CONCLUSION}

The overall work presented here shows that moment based KMeans algorithm can efficiently be used as an image segmentation technique to identify ripe tomatoes. While this experiment is performed on the image of tomatoes, a farmer can choose any crop from the thousands of varieties of crops existing in the world. Therefore this algorithm is not restricted to identification of harvest time of only one crop. One can choose any of the crops.

As future work this algorithm needs to be implemented on a limited device such as a mobile phone to have its full efficiency and effectiveness in the present scenario. After this a farmer will have a handy and automated solution for his problem

\section{REFERENCES}

[1] Margaret H. Dunham," Data mining Introductory and Advanced Topics", pp 10-13, pp 119-159,7 Edition 2011, Pearson
[2] Hu Min, Yang Shuangyuan, "Overview of Image Mining Research", published in The 5th International Conference on Computer Science \& Education Hefei, China. August 24-27, 2010, DOI 978-1-4244-6005-2/10/ (C2010 IEEE

[3] Ashish Phophalia, Suman K. Mitra, Charu Chawla," A Study on Image Segmentation Using Moments ", published in Asian Journal Of Computer Science And Information Technology,Volume 2,Issue 5(2012), ISSN 2249-5126,pp $89-93$.

[4] Piotr Dollár, Zhuowen Tu, Hai Tao and Serge Belongie ,"Feature Mining for Image Classification", published in 2007 IEEE Computer Society Conference on Computer Vision and Pattern Recognition (CVPR 2007), 18-23 June 2007, Minneapolis, Minnesota, USA.

[5] $\mathrm{Hu}$ Min, Yang Shuangyuan, "Overview of Image Mining Research", published in The 5th International Conference on Computer Science \& Education Hefei, China. August 24-27, 2010, DOI 978-1-4244-6005-2/10/ (C)2010 IEEE

[6] G. Polder, G. W. A. M. van der Heijden, I. T. Young, “ Spectral Image Analysis for Measuring ripeness of Tomatoes", published in American Society of Agricultural Engineers (2002), Vol. 45(4): 1155-1161, ISSN 0001-2351

[7] Hassan Asadollahi, Morteza Sabery Kamarposhty, Mir Majid Teymoori ,"Classification and Evaluation of Tomato Images Using Several Classifier", published in 2009 International Association of Computer Science and Information Technology - Spring Conference, 978-07695-3653-8/09 (C) 2009 IEEE DOI 10.1109/IACSITSC. 2009.47

[8] Giuseppe Amato, Paolo Bolettieri, Gabriele Costa, Francesco La Torre, Fabio Martinelli, "Detection of images with adult content for parental control on mobile devices", In Proceedings of the 6th International Conference on Mobile Technology Application Systems Mobility 09 (2009), ISBN 9781605585369:1-5.

[9] Ethem Alpaydin," Introduction To Machine Learning ",The MIT Press Cambridge, Massachusetts London, England, ISBN: 0-262-01211-1 (hc),pp 133-139.

[10] M.K. Hu,"Visual problem recognition by moment invariant",IRE Transaction on Information Theory, Vol IT-8, pp 179-187,Feb 1962. 